\title{
Simplifying differential equations for multi-scale Feynman integrals beyond multiple polylogarithms
}

\author{
Luise Adams, Ekta Chaubey and Stefan Weinzierl \\ PRISMA Cluster of Excellence, Institut für Physik, \\ Johannes Gutenberg-Universität Mainz, D-55099 Mainz, Germany
}

(Dated: February 14, 2017)

\begin{abstract}
In this paper we exploit factorisation properties of Picard-Fuchs operators to decouple differential equations for multi-scale Feynman integrals. The algorithm reduces the differential equations to blocks of the size of the order of the irreducible factors of the Picard-Fuchs operator. As a side product, our method can be used to easily convert the differential equations for Feynman integrals which evaluate to multiple polylogarithms to $\varepsilon$-form.
\end{abstract}

\section{INTRODUCTION}

Precision physics with heavy particles, like the Higgs boson, the top quark or the $W$ - and $Z$-bosons, plays an important part in the current physics programme at the LHC and will become even more important in the upcoming high luminosity runs. Precision physics requires that higher-orders in perturbation theory are taken into account. There is a class of mostly massless processes, where the virtual corrections can be expressed in terms of multiple polylogarithms. Loop integrals can be tackled with differential equations $[1-8]$. We denote by $\varepsilon$ the parameter of dimensional regularisation. If the differential equations can be transformed to an $\varepsilon$-form [7, 8], the solution in terms of multiple polylogarithms is straightforward. However, starting at two-loops, there are integrals which cannot be expressed in terms of multiple polylogarithms. The simplest example is given by the two-loop sunrise integral with internal masses 9 [21], where functions related to elliptic curves occur. In the corresponding system of differential equations one faces the situation, that at order $\varepsilon^{0}$ a system of two coupled differential equations occur, which cannot be transformed away.

If we now look at realistic scattering processes at nextto-next-to-leading order (NNLO) with massive particles it is not unusual that within one topology we have several master integrals, coupled together at order $\varepsilon^{0}$ by the differential equations. We denote by $N$ the number of master integrals within a given topology. For example, for $2 \rightarrow 2$ processes at NNLO topologies with up to 5 master integrals may occur. It would be highly prohibitive, if we had to solve at order $\varepsilon^{0}$ a coupled system of $N$ differential equations. There are indications that topologies with three or more master integrals can be decoupled into blocks of size $2 \times 2$ at worst [16, 22 25]. This raises the question if there is a systematic method which transforms a system into an equivalent system, where at order $\varepsilon^{0}$ the differential equations split into smaller blocks. In this letter we will give an algorithm for this task.

The basic idea is as follows: We first reduce a multiscale problem to a single-scale problem with scale $\lambda$. In a second step we pick a master integral $I$ and deter- mine at order $\varepsilon^{0}$ and modulo sub-topologies the maximal number of independent derivatives $I,(d / d \lambda) I, \ldots$, $(d / d \lambda)^{r-1} I$. This defines a Picard-Fuchs operator of order $r$. For $r<N$ the system decouples into a system of $r$ master integrals and $(N-r)$ master integrals. Let us look at the sector with $r$ master integrals. In a third step we factorise the Picard-Fuchs operator. This will decouple the system into blocks of the size of the order of the irreducible factors of the Picard-Fuchs operator. In a fourth step we reconstruct the multi-variable transformation matrix from the single-variable one.

Although our primary interest are integrals involving elliptic sectors, it should be noted that our approach provides as a side product an algorithm to convert a multiscale system, which has a solution in terms multiple polylogarithms to an $\varepsilon$-form. In this respect it complements other methods 26 33].

\section{THE METHOD}

Let us consider a set of master integrals $I_{1}, \ldots, I_{N}$ depending on kinematic variables $x_{1}, \ldots, x_{n}$. We denote the ordered set of master integrals by the vector $\vec{I}=\left(I_{1}, \ldots, I_{N}\right)$. If the master integrals depend only on a single kinematic variable $x_{1}$, we have a single-scale problem. For two or more kinematic variables $(n \geq 2)$ we have a multi-scale problem. We consider the master integrals in $D=2 m-2 \varepsilon$ space-time dimensions, with $m \in \mathbb{Z}$ and $\varepsilon$ being the dimensional regularisation parameter. Integration-by-parts identities [34, 35] allow us to derive a system of differential equations of Fuchsian type

$$
d \vec{I}=A \vec{I},
$$

where $A$ is a matrix-valued one-form

$$
A=\sum_{i=1}^{n} A_{i} d x_{i} .
$$

The matrix-valued one-form $A$ satisfies the integrability condition

$$
d A-A \wedge A=0 .
$$


We assume that $A$ has an $\varepsilon$-expansion

$$
A=\sum_{j \geq 0} \varepsilon^{j} A^{(j)}=\sum_{i=1}^{n} \sum_{j \geq 0} \varepsilon^{j} A_{i}^{(j)} d x_{i} .
$$

The differential equations are usually solved order by order in $\varepsilon$. A crucial role for solving the system is played by the first term $A^{(0)}$. The higher terms $A^{(j)}$ (with $j \geq 1$ ) only give additional integrations over expressions of lower order. We therefore seek transformations, which simplify $A^{(0)}$. Under a change of basis

$$
\vec{J}=U \vec{I}
$$

one obtains

$$
d \vec{J}=\tilde{A} \vec{J},
$$

where the matrix $\tilde{A}$ is related to $A$ by

$$
\tilde{A}=U A U^{-1}-U d U^{-1} .
$$

The master integrals can be expressed in terms of multiple polylogarithms if there is a transformation $U$ such that $\tilde{A}^{(0)}=0$.

The matrix $A$ has a natural lower block triangular form, which derives from the top topology and its subtopologies, obtained by pinching of propagators. In the following we consider the top topology and we work modulo sub-topologies. The inclusion of sub-topologies leads only to integrations over already determined terms. Let us assume that the top topology has $N$ master integrals. We are in particular interested in the case where no transformation $U$ exists, such that $\tilde{A}^{(0)}=0$. Although it might seem at first sight that we face in this situation at order $\varepsilon^{0}$ a coupled system of $N$ differential equations it is very often the case that the system decouples into blocks of smaller size. In this letter we give a systematic method to decouple the system.

We first reduce the multi-scale problem to a singlescale problem. Let $\alpha=\left[\alpha_{1}: \ldots: \alpha_{n}\right] \in \mathbb{C P}^{n-1}$ be a point in projective space. Without loss of generality we work in the chart $\alpha_{n}=1$. Following [24], we consider a path $\gamma_{\alpha}:[0,1] \rightarrow \mathbb{C}^{n}$, indexed by $\alpha$ and parametrised by a variable $\lambda$. Explicitly, we have

$$
x_{i}(\lambda)=\alpha_{i} \lambda, \quad 1 \leq i \leq n .
$$

We then view the master integrals as functions of $\lambda$. In other words, we look at the variation of the master integrals in the direction specified by $\alpha$. For the derivative with respect to $\lambda$ we have

$$
\frac{d}{d \lambda} \vec{I}=B \vec{I}, \quad B=\sum_{i=1}^{n} \alpha_{i} A_{i} .
$$

The matrix $B$ has again a Taylor expansion in $\varepsilon$ :

$$
B=B^{(0)}+\sum_{j>0} \varepsilon^{j} B^{(j)} .
$$

Let $I$ be one of the master integrals $\left\{I_{1}, \ldots, I_{N}\right\}$. Eq. (9) allows us to express the $k$-th derivative of $I$ with respect to $\lambda$ as a linear combination of the original master integrals. We recall that we work modulo subtopologies. We may even work modulo $\varepsilon$-corrections by using $B^{(0)}$ instead of the full matrix $B$. We then determine the largest number $r$, such that the matrix which expresses $I,(d / d \lambda) I, \ldots,(d / d \lambda)^{r-1} I$ in terms of the original set $\left\{I_{1}, \ldots, I_{N}\right\}$ has full rank. Obviously, we have $r \leq N$. In the case $r<N$ we complement the set $I,(d / d \lambda) I, \ldots,(d / d \lambda)^{r-1} I$ by $(N-r)$ elements $I_{\sigma_{r+1}}, \ldots, I_{\sigma_{N}} \in\left\{I_{1}, \ldots, I_{N}\right\}$ such that the transformation matrix has rank $N$. The elements $I_{\sigma_{r+1}}, \ldots, I_{\sigma_{N}}$ must exist, since we assumed that the set $\left\{I_{1}, \ldots, I_{N}\right\}$ forms a basis of master integrals for this topology. The basis $\left\{I,(d / d \lambda) I, \ldots,(d / d \lambda)^{r-1} I, I_{\sigma_{r+1}}, \ldots, I_{\sigma_{N}}\right\}$ decouples the system into a block of size $r$, which is closed under differentiation at order $\varepsilon^{0}$ modulo sub-topologies and a remaining sector of size $(N-r)$.

Let us now investigate under which conditions the block of size $r$ can be decomposed further. We recall that $r$ is the largest number such that $I,(d / d \lambda) I, \ldots,(d / d \lambda)^{r-1} I$ are independent. It follows that $(d / d \lambda)^{r} I$ can be written as a linear combination of $I,(d / d \lambda) I, \ldots,(d / d \lambda)^{r-1} I$. This defines the Picard-Fuchs operator $L_{r}$ for the master integral $I$ with respect to $\lambda$ :

$$
L_{r} I=0, \quad L_{r}=\sum_{k=1}^{r} R_{k} \frac{d^{k}}{d \lambda^{k}},
$$

where the coefficients $R_{k}$ are rational functions in $\lambda$ and we use the normalisation $R_{r}=1$. Note that the zero on the right-hand side of eq. (11) is understood modulo sub-topologies and modulo terms of order $\varepsilon$. Using always $B^{(0)}$ instead of $B$ ensures that $L_{r}$ is independent of $\varepsilon$. The Picard-Fuchs operator is easily obtained by a transformation to the basis $I,(d / d \lambda) I, \ldots,(d / d \lambda)^{r-1} I$. In this basis the $r \times r$-matrix $\tilde{B}$ has the form

$$
\left(\begin{array}{ccccc}
0 & 1 & \ldots & 0 & 0 \\
& & \ldots & & \\
0 & 0 & \ldots & 0 & 1 \\
-R_{0} & -R_{1} & \ldots & -R_{r-2} & -R_{r-1}
\end{array}\right) .
$$

It is very often the case that the operator $L_{r}$ factorises [6]:

$$
L_{r}=L_{1, r_{1}} L_{2, r_{2}} \ldots L_{s, r_{s}},
$$

where $L_{i, r_{i}}$ denotes a differential operator of order $r_{i}$. Clearly, we have $r_{1}+\ldots+r_{s}=r$. The factorisation in eq. (13) can be obtained with the help of standard computer algebra systems. For example, Maple offers the command "DFactor". The factorisation in eq. (13) can be used to convert the system of differential equations at order $\varepsilon^{0}$ into a block triangular form with blocks of size $r_{1}, r_{2}, \ldots, r_{s}$. A basis for block $i$ is given by

$$
J_{i, j}=\frac{d^{j-1}}{d \lambda^{j-1}} L_{i+1, r_{i+1}} \ldots L_{s, r_{s}} I, \quad 1 \leq j \leq r_{i} .
$$


Let us denote $\vec{J}=\left(J_{1,1}, \ldots, J_{1, r_{1}}, J_{2,1}, \ldots, J_{s, r_{s}}\right)$. Expressing the elements of $\vec{J}$ in terms of the original integrals $\vec{I}$ defines a transformation matrix

$$
\vec{J}=V \vec{I}
$$

$V$ is a function of the parameters $\alpha$ and of $\lambda$ :

$$
V=V\left(\alpha_{1}, \ldots, \alpha_{n-1}, \lambda\right)
$$

We recall that we work in the chart $\alpha_{n}=1$. Setting

$$
U=V\left(\frac{x_{1}}{x_{n}}, \ldots, \frac{x_{n-1}}{x_{n}}, x_{n}\right)
$$

gives the transformation in terms of the original variables $x_{1}, \ldots, x_{n}$. Let us mention that there might be terms in the original $A$, which map to zero in $B$ for the class of paths considered in eq. (8). These terms are derivatives of functions being constant on lines through the origin. An example is given by

$$
d \ln Z\left(x_{1}, \ldots, x_{n}\right),
$$

where $Z\left(x_{1}, \ldots, x_{n}\right)$ is a rational function in $\left(x_{1}, \ldots, x_{n}\right)$ and homogeneous of degree zero in $\left(x_{1}, \ldots, x_{n}\right)$. On the one hand these terms don't contribute if we integrate the differential equation along the paths of eq. (8). On the other hand, these terms are in many cases easily removed by a subsequent transformation.

\section{THE CASE OF LINEAR FACTORS}

Let us consider the special case, where the PicardFuchs operator $L_{r}$ factorises completely into linear factors:

$$
L_{r}=L_{1,1} L_{2,1} \ldots L_{r, 1},
$$

with

$$
L_{i, 1}=\frac{d}{d \lambda}+R_{i, 0}
$$

$R_{i, 0}$ is a rational function in $\lambda$. Then it is possible to construct a transformation such that $\tilde{A}^{(0)}=0$. We first set

$$
J_{i, 1}=\exp \left(\int^{\lambda} d \tilde{\lambda} R_{i, 0}\right) L_{i+1,1} \ldots L_{r, 1} I,
$$

This transforms the system to a form

$$
\frac{d}{d \lambda} \vec{J}=\tilde{B} \vec{J}
$$

where the $\varepsilon^{0}$-term $\tilde{B}^{(0)}$ is lower triangular with zeros on the diagonal. The possible non-zero entries in the lower

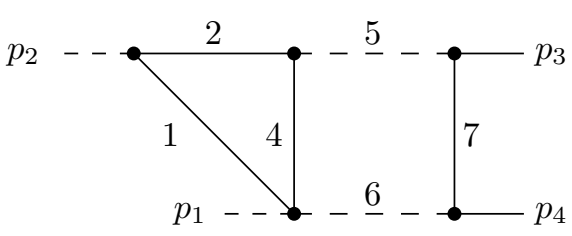

FIG. 1: A two-loop four-point integral with six propagators.

triangle of $\tilde{B}^{(0)}$ are easily removed. It is sufficient to discuss the case of a $2 \times 2$-matrix: For

$$
\tilde{B}^{(0)}=\left(\begin{array}{cc}
0 & 0 \\
N & 0
\end{array}\right)
$$

the transformation

$$
\tilde{V}^{(0)}=\left(\begin{array}{ll}
1 & 0 \\
f & 1
\end{array}\right)
$$

with

$$
f=-\int^{\lambda} d \tilde{\lambda} N
$$

removes the term in the lower left corner. The function $N$ has a partial fraction decomposition in $\lambda$ and we use this technique to remove all terms which are polynomials in $\lambda$ or poles of order 2 and higher. Single poles integrate to logarithms and indicate that our basis elements have non-uniform weight. These are removed by rescaling the master integrals by $\varepsilon$-dependent pre-factors, such that the master integrals have uniform weight. In summary, this gives an easy method to convert a system, where every Picard-Fuchs operator factorises into linear factors, into $\varepsilon$-form.

\section{EXAMPLES}

Let us now look at a few examples. As a first example we consider a two-loop four-point integral with six propagators, shown in fig. (1). Internal solid lines correspond to a mass $m$, dashed lines to mass zero. The external momenta are on-shell, $p_{1}^{2}=p_{2}^{2}=0$ and $p_{3}^{2}=p_{4}^{2}=m^{2}$. The Mandelstam variables are defined by $s=\left(p_{1}+p_{2}\right)^{2}$ and $t=\left(p_{2}+p_{3}\right)^{2}$. The integral depends on two dimensionless variables $x_{1}$ and $x_{2}$, which we may choose as [36 39

$$
s=-m^{2} \frac{\left(1-x_{1}\right)^{2}}{x_{1}}, \quad t=-m^{2} x_{2} .
$$

The internal propagators are labelled by numbers as shown in fig. (11). We denote by $I_{\nu_{1} \nu_{2} \nu_{3} \nu_{4} \nu_{5} \nu_{6} \nu_{7}}$ the scalar 
integral, where propagator $j$ occurs to the power $\nu_{j}$. The seven indices refer to a double-box integral with seven propagators. We use the convention that a scalar propagator is given by $1 /\left(-q_{j}^{2}+m_{j}^{2}\right)$. We use the program "Reduze" 40, 41] to obtain an initial set of master integrals together with the corresponding system of differential equations. The integral in fig. (1) has an elliptic sub-topology, obtained by pinching propagators 2,5 and 6. The topology in fig. (11) has two master integrals, which we may take as

$$
\vec{I}=\left((1+2 \varepsilon) I_{1101111}, I_{1101211}\right) .
$$

The pre-factor $(1+2 \varepsilon)$ in front of the first master integrals ensures that only $A^{(0)}$ and $A^{(1)}$ appear in the $\varepsilon$-expansion of $A$. The Picard-Fuchs operator for $I=(1+2 \varepsilon) I_{1101111}$ is of order 2 and factorises into linear factors:

$$
\begin{aligned}
L_{2} & = \\
& \left(\frac{d}{d \lambda}+\frac{1}{\lambda+1}+\frac{2 \alpha_{1}}{\alpha_{1} \lambda-1}+\frac{2 \alpha_{1} \lambda}{\alpha_{1} \lambda^{2}+1}-\frac{2 \alpha_{1} \lambda}{\alpha_{1} \lambda^{2}-1}\right) \\
& \times\left(\frac{d}{d \lambda}-\frac{1}{\lambda}+\frac{1}{\lambda+1}+\frac{2 \alpha_{1}}{\alpha_{1} \lambda-1}\right) .
\end{aligned}
$$

We may therefore transform to a basis, where $\tilde{B}^{(0)}$ is lower triangular with zeros on the diagonal. The entry on the lower left corner of $\tilde{B}^{(0)}$ has only single poles and is removed by rescaling the first master integral with $(1+2 \varepsilon)$ and the second master integral by $\varepsilon$. This converts the system with respect to the variable $\lambda$ to $\varepsilon$-form. Going back to the original variables we find

$$
\tilde{A}=\left(\begin{array}{ll}
1 & 0 \\
0 & 1
\end{array}\right) d \ln \left(\frac{x_{1}}{x_{2}}\right)+\varepsilon \tilde{A}^{(1)} .
$$

The $\varepsilon^{0}$-term is easily removed by multiplying both master integrals by $x_{2} / x_{1}$. In summary we find that with

$$
\begin{gathered}
U=\left(\begin{array}{cc}
U_{11} & -\frac{(1+2 \varepsilon)\left(x_{1}-1\right)^{3}\left(x_{2}+1\right)^{2}}{2 x_{1}\left(x_{1}+1\right)} \\
\frac{\varepsilon\left(x_{2}+1\right)\left(x_{1}-1\right)^{2}}{x_{1}} & 0
\end{array}\right), \\
U_{11}=\frac{(1+2 \varepsilon)\left(x_{1}-1\right)\left(x_{2}^{2} x_{1}+x_{2} x_{1}^{2}+x_{2}-x_{1}^{2}+3 x_{1}-1\right)}{2 x_{1}\left(x_{1}+1\right)}
\end{gathered}
$$

the transformed system is given by

$$
\begin{aligned}
\tilde{A}= & \varepsilon\left[\left(\begin{array}{ll}
2 & 0 \\
0 & 0
\end{array}\right) d \ln \left(x_{1}+1\right)-\left(\begin{array}{ll}
2 & 0 \\
0 & 2
\end{array}\right) d \ln \left(x_{1}-1\right)\right. \\
& -\left(\begin{array}{ll}
0 & 0 \\
0 & 2
\end{array}\right) d \ln \left(x_{2}+1\right)+\left(\begin{array}{rr}
0 & 0 \\
-1 & 1
\end{array}\right) d \ln \left(x_{1}+x_{2}\right) \\
& \left.+\left(\begin{array}{ll}
0 & 0 \\
1 & 1
\end{array}\right) d \ln \left(x_{1} x_{2}+1\right)\right] .
\end{aligned}
$$

We see that this topology can be transformed to $\varepsilon$-form and does not introduce new elliptic integrations.

Let us now look at a more involved example. We consider the two-loop four-point integral with five propagators shown in fig. (2). The kinematics is as in our first

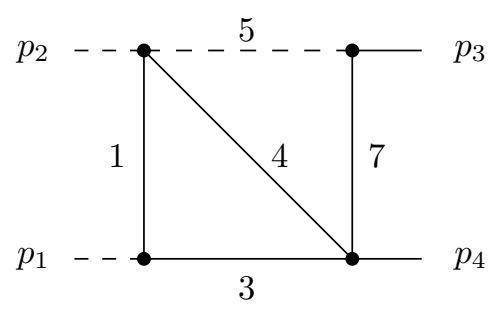

FIG. 2: A two-loop four-point integral with five propagators.

example. This topology has five master integrals. As our initial basis we take

$$
\vec{I}=\left(\varepsilon I_{1011101}, I_{2011101}, I_{1021101}, I_{1012101}, I_{1011201}\right) .
$$

Multiplying the first master integral by $\varepsilon$ ensures that only $A^{(0)}$ and $A^{(1)}$ appear in the $\varepsilon$-expansion of $A$. For the specific system under consideration this also decouples the first master integral $I_{1}=\varepsilon I_{1011101}$ at order $\varepsilon^{0}$ from the remaining ones. We therefore have to consider only a $4 \times 4$-system. Let us pick $I_{2}=I_{2011101}$. Working modulo $\varepsilon$-terms, we find that already the third derivative of $I_{2}$ can be expressed as a linear combination of the lower ones. Adding $I_{5}=I_{1011201}$ to $I_{2},(d / d \lambda) I_{2},(d / d \lambda)^{2} I_{2}$ will give a transformation matrix of full rank. This decouples $I_{5}$ from the $3 \times 3$-system formed by $I_{2},(d / d \lambda) I_{2},(d / d \lambda)^{2} I_{2}$. The Picard-Fuchs operator for $I_{2}$ is therefore of order 3 . It factorises into a second-order operator and a first-order operator:

$$
L_{3}=L_{2} L_{1} .
$$

This decouples the $3 \times 3$-system into a $2 \times 2$-system and a $1 \times 1$-system. The $2 \times 2$-system is irreducible. When lifting the result from the single-scale case to the multiscale case with the variables $\left\{x_{1}, x_{2}\right\}$ we again perform an additional transformation, which removes $d \ln \left(x_{1} / x_{2}\right)$ terms. In summary we are able to decompose the five master integrals for this topology at order $\varepsilon^{0}$ in blocks of size

$$
1,2,1,1 \text {. }
$$

The explicit expressions are longer, however we may display the structure of $\tilde{A}$. We have

$$
\tilde{A}=\left(\begin{array}{ccccc}
0 & 0 & 0 & 0 & 0 \\
0 & * & * & 0 & 0 \\
0 & * & * & 0 & 0 \\
0 & * & * & 0 & 0 \\
0 & * & * & 0 & 0
\end{array}\right)+\varepsilon \tilde{A}^{(1)}
$$

where $*$ indicates a non-zero entry. In this example we see that $A^{(0)}$ cannot be transformed to zero. We find an irreducible $2 \times 2$-system at order $\varepsilon^{0}$. However, we 
achieved to simplify the original $5 \times 5$-system to smaller blocks.

In addition we have applied our method successfully to all sectors of the seven-propagator double-box integral, including the top sector with seven propagators. This sector has five master integrals and decouples into blocks of size $1,2,1$ and 1 .

\section{CONCLUSIONS}

In this letter we presented an algorithm to simplify differential equations for multi-scale Feynman integrals. We first reduced the problem to a single-scale problem and exploited then factorisation properties of the PicardFuchs operator. This allows us to decouple the system at order $\varepsilon^{0}$ into blocks of the sizes of the irreducible factors of the Picard-Fuchs operator. We expect this technique to be useful for precision calculations. A particular special case is given when all Picard-Fuchs operators factorise into linear factors. In this case our method provides an easy algorithm to convert a multi-scale differential system into $\varepsilon$-form.

\section{Acknowledgements}

L.A. and E.C. are grateful for financial support from the research training group GRK 1581.

[1] A. V. Kotikov, Phys. Lett. B254, 158 (1991).

[2] A. V. Kotikov, Phys. Lett. B267, 123 (1991).

[3] E. Remiddi, Nuovo Cim. A110, 1435 (1997), hepth/9711188.

[4] T. Gehrmann and E. Remiddi, Nucl. Phys. B580, 485 (2000), hep-ph/9912329.

[5] M. Argeri and P. Mastrolia, Int. J. Mod. Phys. A22, 4375 (2007), arXiv:0707.4037.

[6] S. Müller-Stach, S. Weinzierl, and R. Zayadeh, Commun.Math.Phys. 326, 237 (2014), arXiv:1212.4389.

[7] J. M. Henn, Phys. Rev. Lett. 110, 251601 (2013), arXiv:1304.1806.

[8] J. M. Henn, J. Phys. A48, 153001 (2015), arXiv:1412.2296.

[9] D. J. Broadhurst, J. Fleischer, and O. Tarasov, Z.Phys. C60, 287 (1993), arXiv:hep-ph/9304303.

[10] M. Caffo, H. Czyz, S. Laporta, and E. Remiddi, Nuovo Cim. A111, 365 (1998), arXiv:hep-th/9805118.

[11] S. Laporta and E. Remiddi, Nucl. Phys. B704, 349 (2005), hep-ph/0406160.
[12] B. A. Kniehl, A. V. Kotikov, A. Onishchenko, and O. Veretin, Nucl. Phys. B738, 306 (2006), arXiv:hep$\mathrm{ph} / 0510235$.

[13] S. Müller-Stach, S. Weinzierl, and R. Zayadeh, Commun. Num. Theor. Phys. 6, 203 (2012), arXiv:1112.4360.

[14] L. Adams, C. Bogner, and S. Weinzierl, J. Math. Phys. 54, 052303 (2013), arXiv:1302.7004.

[15] L. Adams, C. Bogner, and S. Weinzierl, J. Math. Phys. 55, 102301 (2014), arXiv:1405.5640.

[16] L. Adams, C. Bogner, and S. Weinzierl, J. Math. Phys. 56, 072303 (2015), arXiv:1504.03255.

[17] L. Adams, C. Bogner, and S. Weinzierl, J. Math. Phys. 57, 032304 (2016), arXiv:1512.05630.

[18] S. Bloch and P. Vanhove, J. Numb. Theor. 148, 328 (2015), arXiv:1309.5865.

[19] S. Bloch, M. Kerr, and P. Vanhove, Compos. Math. 151, 2329 (2015), arXiv:1406.2664.

[20] S. Bloch, M. Kerr, and P. Vanhove, (2016), arXiv:1601.08181.

[21] E. Remiddi and L. Tancredi, Nucl.Phys. B880, 343 (2014), arXiv:1311.3342.

[22] E. Remiddi and L. Tancredi, Nucl. Phys. B907, 400 (2016), arXiv:1602.01481.

[23] L. Adams, C. Bogner, A. Schweitzer, and S. Weinzierl, J. Math. Phys. 57, 122302 (2016), arXiv:1607.01571.

[24] R. Bonciani et al., JHEP 12, 096 (2016), arXiv:1609.06685.

[25] A. von Manteuffel and L. Tancredi, (2017), arXiv:1701.05905.

[26] T. Gehrmann, A. von Manteuffel, L. Tancredi, and E. Weihs, JHEP 06, 032 (2014), arXiv:1404.4853.

[27] M. Argeri et al., JHEP 03, 082 (2014), arXiv:1401.2979.

[28] R. N. Lee, JHEP 04, 108 (2015), arXiv:1411.0911.

[29] M. Prausa, (2017), arXiv:1701.00725.

[30] O. Gituliar and V. Magerya, (2017), arXiv:1701.04269.

[31] C. Meyer, (2016), arXiv:1611.01087.

[32] L. Tancredi, Nucl. Phys. B901, 282 (2015), arXiv:1509.03330.

[33] J. Ablinger et al., Comput. Phys. Commun. 202, 33 (2016), arXiv:1509.08324.

[34] F. V. Tkachov, Phys. Lett. B100, 65 (1981).

[35] K. G. Chetyrkin and F. V. Tkachov, Nucl. Phys. B192, 159 (1981).

[36] J. Fleischer, A. V. Kotikov, and O. L. Veretin, Nucl. Phys. B547, 343 (1999), hep-ph/9808242.

[37] A. Kotikov, J. H. Kuhn, and O. Veretin, Nucl. Phys. B788, 47 (2008), arXiv:hep-ph/0703013.

[38] R. Bonciani, G. Degrassi, and A. Vicini, Comput. Phys. Commun. 182, 1253 (2011), arXiv:1007.1891.

[39] J. M. Henn and V. A. Smirnov, JHEP 11, 041 (2013), arXiv:1307.4083.

[40] C. Studerus, Comput. Phys. Commun. 181, 1293 (2010), arXiv:0912.2546.

[41] A. von Manteuffel and C. Studerus, (2012), arXiv:1201.4330. 